\title{
Entre la polarización y la catarsis: Nuevas directrices del comportamiento electoral en Estados Unidos
}

\author{
Between Polarization and Catharsis: New Guidelines for Voting Behavior \\ in the United States
}

\author{
José Antonio MEYER RODRíGUEZ \\ Benemérita Universidad Autónoma de Puebla \\ joseantonio.meyer@gmail.com
}

Recibido: 12 de enero del 2017

Aceptado: 17 de marzo de 2017

\begin{abstract}
Resumen
Esta investigación constituye una propuesta inicial que busca contribuir a una mayor comprensión de fenómenos complejos de opinión pública en procesos políticos de alto nivel de polarización social. El planteamiento señala que los estudios sobre comportamiento electoral deben realizarse en forma integral y no solamente considerar el ambiente político, la coyuntura particular o la intermediación mediática, sino también la interacción en redes virtuales e instancias de socialización, el perfil de los candidatos, el valor simbólico de sus discursos y la relatividad de las predicciones demoscópicas. En ese sentido, se identifica que la significativa configuración del candidato Donal Trump en las elecciones presidenciales 2016 en Estados Unidos lo convirtió en un sujeto de identificación pertinente para las aspiraciones y valores de grupos segmentados de población. Sus discursos polarizantes incidieron en la construcción de interpretaciones mayormente emotivas, con componentes convenientes de racionalidad que le dieron sentido a la decisión electoral. Del mismo modo, mediante una intensa actividad en redes de interacción virtual y una movilización social de grupos conservadores, generó una mayor variabilidad perceptiva que incidió en el resultado final de estados del medio oeste y sureste del territorio.
\end{abstract}

\section{Abstract}

This paper is an initial analysis that seeks to contribute to understanding a complex phenomena in public opinion and political processes of high level social polarization. It 
maintains that studies on electoral behavior must be carried out in a comprehensive manner, and not only consider the political environment or the situation of media intermediation, but also the interaction carried out in virtual networks and instances of socialization, the distinction of the candidates, the symbolic value of speeches and the relativity of demoscopic predictions. In this sense, it is identified that the significant configuration of the candidate Donal Trump in the 2016 presidential elections in the United States, made him a relevant identification subject for the aspirations and values of segmented population groups. His polarizing speeches influenced the construction of mostly emotional interpretations, with convenient components of rationality that gave meaning to the electoral decision. In the same way, through intense activity in virtual interaction networks and a social mobilization of conservative groups, it generated greater perceptual variability that influenced the final result of states in the Midwest and Southeast.

Palabras clave: Comportamiento electoral, discurso político, razones de voto, encuestas electorales y redes virtuales.

Key Words: Electoral Behavior, Political speeches, Voting reasons, Electoral surveys and Virtual networks.

\section{Introducción}

Aunque para muchos especialistas en Estados Unidos las predicciones demoscópicas y de los medios de comunicación durante la campaña presidencial de 2016 permitían considerar con cierto sustento ${ }^{1}$ - un mayor nivel de preferencia ciudadana hacia la candidata del Partido Demócrata para ganar la elección, la inercia multiplicadora de rechazo electoral por parte de grupos blancos conservadores, algunos latinos y afroamericanos en estados del medio oeste, sur y sureste del territorio permitió al candidato del Partido Republicano alcanzar el triunfo en una competida elección por un mayor número de votos electorales (The American Presidency Project, 2016). Este resultado generó nuevamente un amplio debate político, mediático y académico sobre la eficacia de las encuestas de opinión como mecanismos de previsión certera del comportamiento electoral en procesos altamente competidos, así como

\footnotetext{
${ }^{1}$ Para las principales encuestadoras del país las predicciones a nivel nacional y local favorecieron en todo momento a la candidata Demócrata Hillary Clinton. Sin embargo, como se demostró tiempo después, muchas de las encuestas locales fallaron en su predicción al no considerar la importancia de los votos indecisos que como en diversas ocasiones terminaron por orientar las decisiones en favor del candidato Republicano en estados clave del centro, sur y sureste del territorio.
} 
el sentido del voto en una sociedad cada vez más segmentada y diversa (AAPOR, 2016). Del mismo modo, destacó la creciente influencia de nuevos componentes de mediación social y tecnológica en la definición de decisiones políticas y electorales (Ward, Welsh \& Smith, 2017).

Cualquier análisis mercadológico de la campaña de la senadora Hillary Clinton podría establecer su pertinencia y eficiencia, con un conjunto de estrategias y medidas prudentes ante las ofensivas del candidato opositor. Sin embargo, por las condiciones propias del proceso y el momento político en ese país, la candidata no consideró la creciente predisposición de diversos grupos sociales a revalorar la significación de los discursos críticos hacia el sistema establecido, incluso grupos conservadores con bajo nivel educativo y mayor edad. Este componente ha sido manifiesto en diferentes experiencias recientes en el mundo que han demostrado la importancia de las expectativas de mejora y evaluaciones del desempeño gubernamental, las cuales definen cada vez más el dilema electoral y establecen los nuevos escenarios de deliberación en los diferentes periodos de campaña. Con base en ello, mientras que el discurso de la candidata Demócrata fue esquemático, repetitivo, convencional, con un corte tradicional, conservador y de poca capacidad movilizadora por factores de género o cambio social, el del candidato Republicano si logró posicionarse gracias a un discurso directo y agresivo que cautivó a muchos electores tradicionales y nuevos votantes que encontraron eco a sus aspiraciones económicas y sociales. La crítica constante a las élites políticas de Washington, la calificación negativa del NAFTA (Tratado de Libre Comercio de Norteamérica), los prejuicios raciales y laborales sobre los inmigrantes latinoamericanos y el cuestionamiento persistente hacia el sistema público de salud impactaron determinantemente el debate en redes virtuales, generaron un efecto en espiral y una identificación gradual con grupos resentidos y esperanzados en nuevas líneas de conducción a nivel presidencial (Diez, BBC News, 2016). De esta manera, al voto blanco y conservador, con diferentes niveles educativos y expectativas de cambio, se agregaron paulatinamente grupos latinos, afroamericanos y nuevos votantes de estados del 
medio oeste y sureste del territorio claramente inconformes con el comportamiento público de la senadora Demócrata (Faus, Periódico El País, 2016). Ello coadyuvó al escalonamiento gradual del triunfo electoral de un candidato, que, si bien tenía poca vinculación con los partidos tradicionales, manifestaba un nacionalismo económico renovado, era evidente su selectividad social y, sobre todo, su preponderancia de los valores estadounidenses sustentados en la fuerza y la supremacía mundial.

Es de destacar que un factor importante en el resultado final de esta elección fue el nivel de participación, el cual fue inferior al 62\% (137 millones 500 mil personas). Ello representó, según el Pew Research Center (2016), una proporción similar a la de 2012 (proceso de reelección) pero poco inferior al 63.6\% de 2008 (proceso inicial de elección). Este inercial nivel de participación si bien manifestó el desencanto ciudadano y decepción con diversas políticas implementadas por la administración de Barack Obama en dos periodos sucesivos, fue también determinado por su propio dilema electoral, el perfil mismo de los candidatos y el impacto de sus discursos y propuestas políticas en los diferentes grupos de votantes. Ante un panorama desalentador, con decremento en el número de votantes afroamericanos y el estancamiento de votantes no blancos, las tendencias mostraron que, a diferencia de 2012, el número de votantes aumentó. En ese sentido, de acuerdo con la Organización Gallup (2016) "[...] la tasa de votantes afroamericanos cayó al 59.6\% después de alcanzar un récord de $66.6 \%$ en 2012 y superar la votación blanca. Es la disminución más grande de votantes de cualquier grupo étnico desde que la participación de votantes blancos cayó del $70.2 \%$ (1992) al 60.7\% (1996). Por su parte, el número de votantes latinos fue un poco menor con un $47.6 \%$ en comparación con las votaciones de 2012 que reportaron 48.0\%. La tasa de participación de votantes asiáticos aumentó a 49.3\% en 2016 frente al 46.9\% de 2012, superando a los hispanos por primera vez desde 1996. Aunque ellos siguen representando una proporción menor que los hispanos, en 2016 votaron alrededor de 5 millones frente a los 3.8 millones de 2012. Los votantes blancos tuvieron un ligero aumento al pasar de $64.1 \%$ en 2012 al 65,5\% en 2016". En ese contexto, la actitud proactiva e irreverente del empresario 
durante la campaña presidencial y la postura conservadora de la senadora Demócrata si resultó relevante para los votantes indecisos y nuevos partícipes en aquellos estados que tradicionalmente se han perfilado como estratégicos para el triunfo final en las elecciones presidenciales.

Otro dato relevante es que a pesar de que Hillary Clinton alcanzó un mayor número de votos populares con 62 millones 523 mil 126 (BBC Mundo, 2016), ellos se concentraron en estados de tradición Demócrata. Sin embargo, perdió 3.5 millones de sufragios en los estados indecisos en comparación con los alcanzados por el candidato Barack Obama en las elecciones de 2012 y 2008. Es decir, aunque mantuvo la supremacía en California, Oregon, Washington, Nuevo Mexico, Colorado, Illinois, Minnesota, Massachusetts, Nueva York y Virginia, fue desplazada por su opositor en los estados del medio oeste y sureste. En términos étnicos el resultado fue todavía más significativo, porque no logró mantener las preferencias de algunos votantes latinos, afroamericanos, independientes y jóvenes que habían votado en su momento por el candidato Demócrata en las dos últimas elecciones presidenciales. Tampoco pudo movilizar a los llamados milenials ${ }^{2}$, altamente identificados con el opositor senador Demócrata Bernie Sanders (Vermont), que suscitaban una expectativa favorable desde el inicio del proceso (Fry, 2017). En contraste, Donald Trump si logró sumar un mayor número de votos que los alcanzados por los candidatos del Partido Republicano en las dos últimas elecciones presidenciales en los estados clave ${ }^{3}$ y aprovechó -como otros candidatos Republicanos en el pasado- el sistema de elección indirecta para

\footnotetext{
${ }^{2}$ Los llamados milenials son las personas nacidas en la última década del siglo XX y que ascendían en 2016 a 69 millones 600 mil personas. Su participación en la elección presidencial fue del $75 \%$, con un voto más o menos equitativo entre ambos candidatos. Sin embargo, su voto no fue significativo en el resultado final de los estados indecisos.

${ }^{3}$ Como los votos alcanzados por Mitt Romney en 2012 (60 millones 933 mil 500 personas) y John McCain en 2008 (59 millones 948 mil 323 personas).
}

Facultad de Ciencias Políticas, Sociales y de la Comunicación Universidad de La Laguna Avenida César Manrique, s/n; Campus de Guajara 38071 La Laguna, Tenerife (Islas Canarias - España) 
sumar un mayor número de delegados y votos en el Colegio Electoral ${ }^{4}$. Además, aunque las predicciones demoscópicas y mediáticas hacia el final de la campaña señalaban a Clinton como la virtual ganadora en Nevada (6 votos electorales) por el voto hispano y resultados muy cerrados en Ohio (18 votos electorales) y Pennsylvania (20 votos electorales), se presagiaban votaciones muy reñidas en La Florida (29 votos electorales), Carolina del Norte (15 votos electorales) ${ }^{5}$, Wisconsin (10 votos electorales) y Michigan (16 votos electorales). Ello a pesar de que se había presentado una supremacía Demócrata durante los últimos años $^{6}$. En la Tabla No. 1 puede observarse un comparativo de votos por elecciones y candidatos en 2008, 2012 y 2016, en la cual son evidentes las diferencias:

\begin{tabular}{|c|c|c|c|c|c|c|}
\hline $\begin{array}{l}\text { Comparaciór } \\
\text { en las últimas el }\end{array}$ & $\begin{array}{l}\text { tos popu } \\
\text { es presid }\end{array}$ & $\begin{array}{r}\text { Tabla } \\
\text { es y electo } \\
\text { ciales en e }\end{array}$ & $\begin{array}{l}\text { No. } 1 \\
\text { cales de lo } \\
\text { tados del }\end{array}$ & $\begin{array}{l}\text { rincipale } \\
\text { dio este }\end{array}$ & $\begin{array}{l}\text { artidos pc } \\
\text { y sur es }\end{array}$ & $\begin{array}{l}\text { cos } \\
\text { del país }\end{array}$ \\
\hline Estados / Votos & Elecció & 2008 & Elecci & n 2012 & Elecció & 2016 \\
\hline & $\begin{array}{c}\text { Obama/ } \\
\text { Biden }\end{array}$ & $\begin{array}{l}\text { McCain/ } \\
\text { Palin }\end{array}$ & $\begin{array}{l}\text { Obama/ } \\
\text { Biden }\end{array}$ & $\begin{array}{l}\text { Romney/ } \\
\text { Ryan }\end{array}$ & $\begin{array}{l}\text { Clinton/ } \\
\text { Kaine }\end{array}$ & $\begin{array}{l}\text { Trump/ } \\
\text { Pence }\end{array}$ \\
\hline Nevada (05 - 06) & 531,884 & 411,988 & 531,373 & 475,064 & $\begin{array}{r}539,260 \\
+27,202\end{array}$ & 512,058 \\
\hline Ohio $(20-18)$ * & $2 ' 708,988$ & 2'502,218 & 2827,709 & 2661,437 & 2268,839 & $\begin{array}{r}2279,543 \\
+10,704\end{array}$ \\
\hline $\begin{array}{l}\text { Pennsylvania (21 - } \\
\text { 20) * }\end{array}$ & 3'192,316 & $2 ` 586,496$ & 2990,274 & 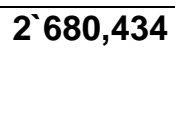 & 29926,441 & $\begin{array}{r}2970,733 \\
+44,292\end{array}$ \\
\hline
\end{tabular}

\footnotetext{
${ }^{4}$ El voto popular favoreció a la senadora Clinton con el $48.03 \%$ de los sufragios contra el $47.3 \%$ del candidato Trump. Sin embargo, en el Colegio Electoral el candidato Republicano alcanzó 306 votos electorales contra 232 de la candidata Demócrata.

${ }^{5}$ Ambos estados de tradición Republicana fueron conquistados en 2008 por el candidato Demócrata Barack Obama, producto de una movilización importante del voto afroamericano y latino.

${ }^{6}$ Esos estados habían sido dominados por el Partido Demócrata durante décadas, aunque en los años ochenta del siglo XX apoyaron los triunfos de los presidentes Republicanos Ronald Reagan (1980 y 1984) y George $\mathrm{H}$. Bush (1988).
}

Facultad de Ciencias Políticas, Sociales y de la Comunicación Universidad de La Laguna 


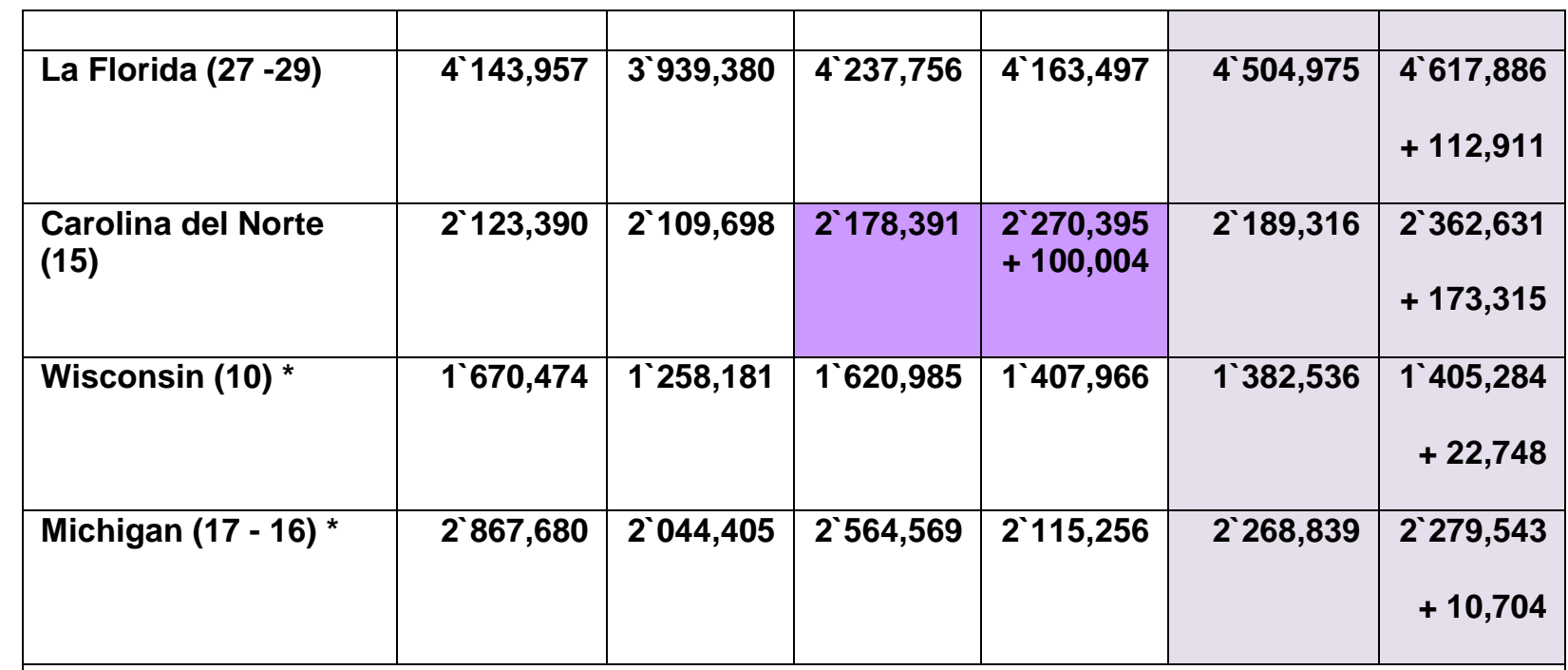

Fuente: Elaboración propia a partir de la información oficial publicada por la Federal Election Commission. Federal Elections 2008. Election Results for the U S President, the U S Senate and U S House Representatives. Washington, D. C. Federal Elections 2012. Election Results for the US President, the U S Senate and U S House Representatives. Washington, D. C. Federal Elections 2016. Election Results for the U S President, the U S Senate and U S House Representatives. Washington, D. C.

En esa condición, aunque muchos analistas calificaron el resultado como inesperado, el análisis detallado de los diversos elementos que intervinieron en el proceso electoral documenta la significación de las variaciones en el comportamiento de los estadounidenses y la preponderancia de los valores tradicionales sobre el pragmatismo y las expectativas sobre resultados no satisfechos. Del mismo modo, las tendencias de participación en esas elecciones demuestran la importancia que para los diferentes grupos de población tiene el perfil de los candidatos, el enfoque movilizador del discurso, las propuestas de campaña, el contexto electoral (elección inicial o reelección), el dilema electoral y el sentido del voto en cada elección particular. Con base en ello, el esquema de la presente investigación plantea la necesidad de realizar un análisis integral del proceso de elección presidencial considerando las siguientes categoría

- Ambiente referencial antes y durante la campaña

- Elementos coyunturales en los discursos de los candidatos 
(dicotomía en la comunicación)

- Interpretaciones de grupos de población indecisos con disposición de voto (percepción selectiva)

- Representaciones sociales como resultado de la interdependencia y globalización (referencias comparativas)

- Formas de deliberación y expresión colectiva en eventos de alto interés público (interpretación social)

- Formación de la opinión pública como resultado de procesos complejos (aproximaciones significativas).

Con base en ello, se establece que ante el desencanto social con el sistema político y el desempeño de los gobiernos la representación simbólica de candidatos de perfil no convencional se ha convertido para sujetos de mayor edad y limitadas posibilidades de mejora económica en un componente de identificación pertinente para su aspiraciones y valores. La intensa actividad en redes de interacción virtual, junto a una dinámica social e institucional más participativa generan un mayor número de instancias mediadoras $\mathrm{y}$, por tanto, mayor variabilidad perceptiva e incertidumbre en la sociedad (Chadwick, 2013) Dichas incertidumbres inciden cada vez más en la construcción de interpretaciones emotivas con componentes básicos de racionalidad (Clementson, 2016). Las encuestas de percepción social tienen cada vez un valor más relativo en la predicción de intenciones de voto, debido a una diversidad mayor de grupos de población, su alta variabilidad de intereses y necesidades (Del Tronco, Flores y Madrigal, 2016). Por tanto, este análisis constituye una consideración inicial y aproximatoria que busca contribuir a la mayor comprensión de fenómenos complejos de opinión pública en procesos políticos de alto nivel de significación política y social. Asume, además, que los estudios sobre comportamiento electoral requieren realizarse en forma integral y no solamente considerar el ambiente político, la coyuntura particular o la intermediación mediática, sino también la interacción en redes virtuales, las dinámicas de 
socialización, la distinción de los candidatos participantes y el valor simbólico de discursos que alteran y relativizan las predicciones de las encuestas demoscópicas.

\section{Metodología de investigación}

Esta investigación establece que el análisis integral de distintos elementos permite identificar las tendencias de reconfiguración creciente en los procesos de representación social durante elecciones altamente competidas. En tal forma, la primera categoría define que el ambiente (económico, político y social) en que se realizan las campañas son muy importantes en la definición del denominado dilema electoral y los resultados mismos de la elección ${ }^{7}$. El estado de ánimo de los electores, sus motivaciones, percepciones, antecedentes de comportamiento y preferencias partidistas son aspectos que influyen determinantemente en las decisiones de los diferentes grupos sociales. Ellos tienen un valor muy significativo en la construcción de percepciones y preferencias, pero, sobre todo, en la conformación del voto final en grupos sociales indecisos y la abstención de grupos independientes y de menor edad.

La segunda categoría manifiesta que la acción continua de los medios de comunicación es una variable importante de intervención y persuasión, pero su efecto tiende a relativizarse para muchas audiencias y su incidencia directa en la percepción ya no es tan sustancial para explicar el comportamiento de los electores. Ello se debe a que en el presente los espacios de interacción virtual de alcance global se expanden con mucha rapidez mientras las redes tradicionales se reconfiguran y adquieren un nuevo rol en los procesos de socialización política. En ese contexto, el valor simbólico de las instancias de mediación social e institucional se restablecen y son tan importante como el de los medios de comunicación

\footnotetext{
${ }^{7}$ Diferentes autores conciben el dilema electoral como un constructo político y social de alto valor simbólico que determina las decisiones de voto de amplios grupos de población.
} 
dada su capacidad de influencia y cercanía con las decisiones finales de los votantes. En el caso de las redes tecnológicas, si bien se destaca la alta dispersión temática con un alto nivel de emotividad y subjetividad argumentativa, también se reconoce su alto potencial para compartir intereses comunes, identificar situaciones similares y realizar catarsis en colectivo que pueden ser muy importantes al momento de tomar decisiones electorales en contextos de incertidumbre y polarización ideológica.

La tercera categoría aprecia la pertinencia de las mediciones de intención de voto en sectores sociales tradicionales, pero también plantea que, en las condiciones actuales de incertidumbre económica y social, con una amplia adjetivación y juicios de valor, reduccionismo en el debate político y emotividad catártica en las expresiones virtuales, su aproximación a los resultados se relativiza y no necesariamente representa las aspiraciones y percepciones de los diversificados grupos ciudadanos. A muchos votantes hoy no les preocupa mucho por qué partido votar sino cómo van a gobernar los candidatos e, incluso, cómo van a incidir directamente en la solución de los problemas económicos y sociales de su región y país. La cuarta categoría destaca que los esquemas discursivos expresados en propuestas políticas y apreciaciones individuales se convierten para los electores en una distinción, en una marca de identificación que se adopta a partir de intereses y aspiraciones individuales y colectivas.

Con base en ellas, la presente investigación plantea la realización de un análisis de los componentes de mayor significación en términos de opinión pública para explicar con mayor pertinencia como se manifestó el comportamiento de grupos sociales específicos en estados de amplia variabilidad durante la elección presidencial estadounidense de 2016. Asimismo, de qué manera se construyeron las intenciones de voto de los grupos más tradicionales, conservadores, con menor educación y origen rural. El propósito es estructurar argumentaciones mayormente sustentadas que superen las especulaciones y contribuyan al estudio de los procesos de formación de opinión pública y configuración de decisiones de 
voto en elecciones determinados por contextos de alta incertidumbre y polarización ideológica.

\section{Resultados de la investigación}

\section{El dilema electoral}

El ambiente político en que se realizaron las precampañas al interior de los partidos mayoritarios mostró dos situaciones contrastantes que determinaron, en gran medida, los resultados finales. Por un lado, a pesar de que para muchos votantes los ocho años continuos del presidente Barack Obama fueron hasta cierto punto bien evaluados ${ }^{8}$, el proyecto de la candidata Hillary Clinton no contó con un posicionamiento político definido por la intensa lucha de contraste con el senador Bernie Sanders que la obligó a realizar diversas negociaciones cupulares para mantener la unidad partidaria previa al proceso de nominación ${ }^{9}$. Por otro, al inicio del proceso preelectoral los analistas presagiaban que las intenciones de voto en favor de un candidato Republicano estarían determinadas por la presencia de personajes de amplia convocatoria. En esa dimensión, el empresario Donald Trump logró con su discurso contrastante deslindarse de las divisiones al interior del Partido Republicano, incorporar a algunos integrantes del Tea Party ${ }^{10}$ a su campaña y constituir una

${ }^{8}$ Para Gallup (2016) el 57\% de los estadounidenses señalaban hacia el final del segundo periodo presidencial de Barack Obama una buena opinión. Un poco más de la mitad consideraba buena o muy buena su administración, aunque dos de cada tres entrevistados consideraba que no cumplió con algunas de sus promesas.

${ }^{9}$ Este candidato demostró la vulnerabilidad de la senadora Clinton y su poca capacidad de respuesta y adaptabilidad ante situaciones críticas. Ello desgastó su candidatura y mostró al opositor la forma más pertinente para atacarla en los debates y descalificar sus discursos.

${ }^{10}$ El Partido del Té se creó por destacados integrantes del Partido Republicano en oposición al gobierno de Barack Obama, con el objeto esencial de recuperar las raíces tradicionales de la democracia estadounidense sustentada en ciudadanos blancos, protestantes y anglosajones. Por su influencia al interior del Partido Republicano, en 2010 lograron que algunos de sus integrantes alcanzaran posiciones importantes en el congreso federal y algunos estados, lo que fortaleció su capacidad para nominar al candidato a la Presidencia. 
candidatura fuerte y de unidad capaz de competir con posibilidades reales por la presidencia del país. Ello quedó de manifiesto con los triunfos ante los exgobernadores Jeb Bush (Florida) y John Kasich (Ohio) y los representantes Mario Rubio (Florida) y Ted Cruz (Texas), a pesar de carecer de una carrera política y una militancia partidista real. Ello le otorgó una amplia atención pública que fue aprovechada para movilizar a diversos grupos sociales y generar una dinámica creciente que lo proyectó como candidato único a la nominación Republicana. En consecuencia, mientras que la campaña del candidato Trump siempre fue en ascenso y alcanzó una movilización social significativa en redes virtuales, la de Clinton fue bastante intermitente y sujeta a cuestionamientos constantes que terminaron por debilitar sus posibilidades de triunfo en estados indecisos por el rechazo de algunos grupos blancos, latinos y afroamericanos.

Durante las elecciones primarias las indagaciones demoscópicas reportaban que para muchos electores temas como el terrorismo, la confrontación permanente del presidente con el Congreso, el incumplimiento de propuestas por parte de la administración Obama y el debate sobre el uso libre de armas (Segunda Enmienda Constitucional) gravitaban en el ánimo social y orientaban las intenciones de voto. Sin embargo, en estados de alto desarrollo industrial la falta de crecimiento económico posterior a la crisis financiera de 2008, la instalación de empresas estadounidenses en territorios extranjeros como resultado de los tratados de libre comercio, el incremento del desempleo entre la población más joven y la reducción de oportunidades de grupos minoritarios en el acceso a las universidades, incidieron importantemente en la postura electoral de distintos grupos de población. A ellas se agregaron variadas inconsistencias de un sistema sanitario en reconstrucción, el resurgimiento de grupos radicales a nivel regional, el rechazo creciente de sectores radicales a la población indocumentada, la falta de consensos para el restablecimiento de relaciones con Cuba y la no restricción del ingreso al territorio de algunos grupos musulmanes.

No obstante que la derrota de Mike Romney en 2012 diluyó su fuerza, mantuvo su vigencia e influencia al interior del Partido Republicano. 
Posterior a las convenciones de nominación partidaria, el candidato Republicano logró maximizar el rendimiento electoral entre los votantes blancos, particularmente los de menor educación y mayor conservadurismo social, mediante una retórica antiinmigrante y muy variados juicios sobre la política exterior del presidente Obama y la intervención de Clinton como Secretaria de Estado en el primer periodo. En ese contexto, sus discursos colmados de emotividad, sobreestimación y sarcasmo, marcado nacionalismo y proteccionismo económico, encontraron el ambiente propicio para fortalecer su aceptación. Adicionalmente, sus posturas alineadas al fanatismo religioso (sobre todo, las del candidato a vicepresidente Mike Pence) en cuestiones como el aborto y la negación de los derechos alcanzados por la comunidad LGBTQ, lograron amplia aceptación en el debate social. La fuerza electoral compactó la base del Partido Republicano a pesar del alejamiento con algunos de sus líderes, lo que incorporó a votantes tradicionales del medio rural y alentó a muchos independientes, como los sectores sindicalizados del noreste del país inconformes con las propuestas de la candidata Demócrata. La optimización de este voto concurrente, aunado a la disminución participativa de electores proclives al Partido Demócrata, fueron determinantes para el resultado final.

En términos específicos, fueron los votos de una población inconforme asentada en los estados de La Florida y Carolina del Norte, al que se agregaron sectores de Wisconsin, Michigan y Pensilvania, los que permitieron a Trump alcanzar la victoria final en el Colegio Electoral. Uno de los últimos candidatos Republicanos ganadores en esos estados había sido Ronald Reagan (1980 y 1985), quien conquistó a los electores con un lenguaje conservador en lo político, comprometido con el nacionalismo económico y una postura férrea contra los enemigos del país y la defensa de la democracia liberal. Es decir, un candidato con un perfil discursivo y un carisma mediático muy parecido al de Donald Trump. Los estados de Michigan y Pensilvania que integran el llamado eje industrial del medio este, al que se agrega Wisconsin con una economía pecuaria industrial, mantenían una importante 
tradición Demócrata por el peso de los sindicatos y porque en sus principales ciudades (Detroit y Marquette, en Michigan, Filadelfia y Pittsburgh, en Pensilvania, o Milwaukee en Wisconsin) se ha producido un tejido social, racial y cultural diverso y cosmopolita, donde el peso de la religión tiende a reducirse y el pensamiento conservador declina y abre paso a ideas más liberales y progresistas. Sin embargo, junto a esos enclaves urbanos, el resto son territorios rurales, con poca diversidad étnica y un profundo conservadurismo religioso. En tal forma, en una misma zona coexisten dos realidades contrastantes. Por un lado, una población mayoritariamente blanca, con un nivel educativo bajo, escasa participación, marcado conservadurismo ideológico e importante orientación religiosa. Por otro, una demografía diversa, progresista, cosmopolita y con una cultura religiosa más tolerante.

El análisis del voto final de los distintos grupos sociales en esos estados identificó el peso de esos componentes en la decisión mayoritaria, a los que se agregaron elementos de coyuntura como los pronunciamientos del director del FBI sobre el comportamiento de Hillary Clinton como Secretaria de Estado (Faus, El País, 2016), la reducción de la dinámica económica en el país y la expansión de las migraciones latinoamericanas hacia nuevos territorios. Hasta la última elección presidencial (2012) el Partido Demócrata había mantenido una conexión directa con la diversidad de los enclaves urbanos y suburbanos de esa zona geográfica, pero su influencia fue disminuyendo entre los sindicatos ante los reclamos no atendidos por revertir los llamados efectos de los tratados de libre comercio. Este fue el único punto de convergencia con el candidato Bernie Sanders, pero no fue considerado en la propuesta de la candidata Clinton a quien se le percibió como conservadora, promotora del libre comercio y poco comprometida con la reconversión industrial del sector automotriz ${ }^{11}$. En este sentido, mientras la Demócrata no supo aprovechar la popularidad alcanzada por el

\footnotetext{
${ }^{11}$ El presidente Obama tuvo una posición proactiva en materia de libre comercio y su pensamiento en casos como el Tratado de la Cuenca del Pacífico fue alcanzar las mejores condiciones laborales y ambientales desde espacios de colaboración, protegiendo también otros intereses como los derechos de propiedad intelectual e industrial y asumir el liderazgo de mercado antes que China.
}

Facultad de Ciencias Políticas, Sociales y de la Comunicación Universidad de La Laguna Avenida César Manrique, s/n; Campus de Guajara 38071 La Laguna, Tenerife (Islas Canarias - España) 
presidente Obama en esa zona del territorio, Trump si supo movilizar a los sectores rurales de Pensilvania con un discurso sobre la supremacía racial y arrebatarle a Clinton el $9 \%$ de su voto tradicional, principalmente ciudadanos blancos, mayores de 45 años de la clase trabajadora que, teniendo empleo o subempleo, buscaron el apoyo gubernamental para la recuperación del sector industrial. El discurso descalificador e injurioso del empresario prometió recuperar los empleos del pasado y transformar la economía estadounidense para alcanzar el voto de esos sectores territoriales. De esta manera, el Periódico The New York Times (2017) reportó que "el mensaje de Trump Make America Great Again [...resultó...] para los sectores suburbanos de Michigan y Pensilvania, una invocación nostálgica, conservadora y de resistencia ante el inminente cambio económico y social. Con ello se revirtió la tendencia electoral de los últimos años, maximizó el voto rural y debilitó el escudo azul de los enclaves urbanos"12.

\section{Redes de interacción virtual}

Para Cellán (BBC News, 2016:1), junto al cuestionamiento persistente de los medios de comunicación más influyentes del país y miembros prominentes del Partido Republicano, el uso estratégico de redes virtuales le permitió a Donald Trump alcanzar una gran notoriedad pública y generar una amplia expectativa en diversos grupos de población. La creación de diferentes comunidades tecnológicas fue por demás determinante en su campaña al dar un impulso fundamental a su propuesta ideológica, aunque ello fuera con modos bastante peligrosos en términos democráticos. En efecto, a diferencia de Barack Obama, quien gestionó con mucha eficacia las redes virtuales para captar fondos y movilizar el voto minoritario e independiente, Trump tuiteó más que cualquier otro candidato presidencial emitiendo una constante de juicios de valor sin mucho sustento, realizando descalificaciones constantes al gobierno y su opositora Demócrata y difundiendo, sobre todo, noticias falsas en

\footnotetext{
12 Esto a pesar de la política de salvamento y apoyo llevada a cabo por la administración Obama durante la bancarrota de la industria automotriz en la ciudad de Detroit.
}

Facultad de Ciencias Políticas, Sociales y de la Comunicación Universidad de La Laguna 
forma de verdades. De acuerdo con Fajardo (BBC News, 2016), este ejercicio libertino del derecho a comunicar cautivó a 4 millones de seguidores en Twitter y 5 millones más en Facebook que Hillary Clinton. Asimismo, con el entusiasta ánimo de usuarios por el contenido publicado, este candidato logró incrementar sus preferencias y votos en muchos estados conservadores y resentidos con el gobierno Demócrata de los últimos 8 años. Del mismo modo, según datos de la compañía Social Flow (2016), "Trump tuvo tres veces más exposición gratuita en redes sociales que Hillary Clinton lo que lo convirtió rápidamente en la persona de la que más se habló en el planeta". El propio Trump reconoció en una entrevista con el diario The New York Times (Barbaro, 2016:5) que "antes de las redes sociales, la única opción era demandar a sus rivales, pero con la moderna habilidad para discutir en las plataformas virtuales se tiene más poder que los demás [...]. En esta forma, las redes sociales son más poderosas que el dinero" ${ }^{13}$. Con esta estrategia, el candidato libró el filtro y la censura de los medios de comunicación tradicionales y le habló de manera directa e insistente a los ciudadanos sobre sus temas de mayor interés mediante instrumentos instantáneos de amplia capacidad de diseminación, estableciendo las bases de un nuevo patrón comunicacional durante las campañas electorales. ${ }^{14}$

Este uso de las redes virtuales durante la campaña presidencial de 2016 ratificó el surgimiento de la llamada era del post-truth (post-verdad), la cual permite a los candidatos burlar los tradicionales fact checkers o gatekeepers (reporteros y moderadores de debates) y comunicarse directamente con los ciudadanos. En esa condición, según PolitiFact (Drobnic,

\footnotetext{
${ }^{13}$ De hecho, Trump gastó en su campaña casi la mitad de lo que Clinton invirtió para movilizar el voto a su favor. Lo que constituye un nuevo paradigma en la historia electoral de Estados Unidos. Antes las elecciones presidenciales se ganaban con dinero, ahora con una gestión estratégica de medios alternativos, los cuales además están fuera de toda regulación.

${ }^{14}$ Durante la elección presidencial de 2008 Obama alcanzó 2 millones 379 mil 102 seguidores en comparación con los 620 mil 359 del senador Republicano John McCain. Adicionalmente, diferentes estudios han demostrado que los políticos opositores en el congreso federal tienden hoy a usar cada vez más las redes virtuales que sus contrapartes como un instrumento de interacción directa con el público.
}

Facultad de Ciencias Políticas, Sociales y de la Comunicación Universidad de La Laguna 
2016), "solo el $14 \%$ de los pronunciamientos de Trump fueron verdaderos, pero inundaron los espacios con cuentas falsas que fueron ampliamente replicadas por sus tuits, aunque algunas veces fueron muy poco apropiadas y éticamente correctas en una campaña presidencial". La página de internet Twitter Audit (2016), por su parte, destacó que el 39\% de los seguidores de Trump fueron robots generados por una computadora. Con base en ello, Por ello, Rodríguez (2018) señaló que "las redes sociales están profundizando la brecha entre los estadounidenses más conservadores y los más liberales, ya que los usuarios de redes virtuales viven en una burbuja de filtro en la que las plataformas adaptan el contenido según sus intereses, alejándonos de otros puntos de vista".

Beltrán (2017:108) ha asegurado que la virtualidad inducida explica en cierta medida por qué los seguidores de Clinton estaban tan sorprendidos con los resultados finales de la elección. Ello se debió a que "hoy en día la mayoría de los estadounidenses no creen en la existencia del otro si no lo encuentran en su canal de noticias de Facebook. Asimismo, muy a menudo la gente no puede entablar conversaciones productivas con personas que tienen opiniones diferentes porque el diálogo en las redes se ha vuelto virulento". En ese contexto, un estudio del Pew Research Center (2017) halló que "el $84 \%$ de los usuarios de redes concuerdan en que la gente dice cosas cuando discute sobre política que nunca se atrevería a decir en persona".

\section{Sondeos de opinión}

Los análisis realizados por los especialistas de la AAPOR (Asociación Americana de Investigadores de Opinión Pública), reportaron que algunas de las inconsistencias entre las proyecciones previas de las encuestadoras y los resultados de las encuestas de salida se debieron a que no registraron cambios importantes en las intenciones de voto durante los últimos días de la campaña. De igual forma, que el impacto de las investigaciones de la FBI a la ex Secretaria de Estado tuvo un efecto más negativo de lo identificado en la percepción de 
los electores latinos, afroamericanos y nuevos votantes quienes decidieron variar finalmente su decisión de votar o no votar. Estas particularidades locales y su tendencia escalonada hacia el final del proceso son fundamentales para cualquier tipo de análisis del comportamiento electoral de los estadounidenses pero, sobre todo, muestran la reñida contienda escenificada por ambos candidatos. En la Tabla No. 2 se identifica la significación de las distintas segmentaciones y su impacto final en las decisiones electorales de los distintos grupos de población:

\begin{tabular}{|c|c|c|c|c|}
\hline \multicolumn{5}{|c|}{$\begin{array}{c}\text { Tabla No. } 2 \\
\text { Razones de voto de los ciudadanos durante la elección presidencial } 2016 \text { en Estados } \\
\text { Unidos }\end{array}$} \\
\hline Categoría & Total & Hillary Clinton & Donald Trump & Otros \\
\hline \multicolumn{5}{|c|}{ Género } \\
\hline Hombres & $47 \%$ & $41 \%$ & $52 \%$ & $7 \%$ \\
\hline Mujeres & $53 \%$ & $54 \%$ & $41 \%$ & $5 \%$ \\
\hline \multicolumn{5}{|c|}{ Edad } \\
\hline 18-24 años & $10 \%$ & $56 \%$ & $34 \%$ & $10 \%$ \\
\hline 25-29 años & $9 \%$ & $54 \%$ & $38 \%$ & $8 \%$ \\
\hline 30-39 años & $17 \%$ & $51 \%$ & $39 \%$ & $10 \%$ \\
\hline 40-49 años & $19 \%$ & $46 \%$ & $49 \%$ & $5 \%$ \\
\hline 50-64 años & $30 \%$ & $44 \%$ & $52 \%$ & $4 \%$ \\
\hline 65 años o más & $16 \%$ & $45 \%$ & $52 \%$ & $3 \%$ \\
\hline \multicolumn{5}{|c|}{ Género / Edad / Mujeres } \\
\hline $18-29$ años & $19 \%$ & $55 \%$ & $36 \%$ & $9 \%$ \\
\hline 30-44 años & $25 \%$ & $51 \%$ & $41 \%$ & $8 \%$ \\
\hline 45-64 años & $40 \%$ & $44 \%$ & $52 \%$ & $6 \%$ \\
\hline 65 años o más & $16 \%$ & $45 \%$ & $52 \%$ & $3 \%$ \\
\hline \multicolumn{5}{|c|}{ Género / Edad / Hombres } \\
\hline $18-44$ & $44 \%$ & $53 \%$ & $39 \%$ & $8 \%$ \\
\hline 45 años o más & $56 \%$ & $44 \%$ & $52 \%$ & $4 \%$ \\
\hline \multicolumn{5}{|c|}{ Etnia } \\
\hline Blancos & $71 \%$ & $37 \%$ & $57 \%$ & $6 \%$ \\
\hline Afroamericanos & $12 \%$ & $89 \%$ & $8 \%$ & $3 \%$ \\
\hline Latinos & $11 \%$ & $66 \%$ & $28 \%$ & $6 \%$ \\
\hline Asiáticos & $4 \%$ & $65 \%$ & $27 \%$ & $8 \%$ \\
\hline Otra & $3 \%$ & $56 \%$ & $36 \%$ & $8 \%$ \\
\hline \multicolumn{5}{|c|}{ Etnia / Género } \\
\hline Hombres blancos & $34 \%$ & $31 \%$ & $62 \%$ & $7 \%$ \\
\hline Mujeres blancas & $37 \%$ & $43 \%$ & $52 \%$ & $5 \%$ \\
\hline Hombres afroamericanos & $5 \%$ & $82 \%$ & $13 \%$ & $5 \%$ \\
\hline Mujeres afroamericanas & $7 \%$ & $94 \%$ & $4 \%$ & $2 \%$ \\
\hline
\end{tabular}

Facultad de Ciencias Políticas, Sociales y de la Comunicación Universidad de La Laguna 


\begin{tabular}{|c|c|c|c|c|}
\hline Hombres latinos & $5 \%$ & $63 \%$ & $32 \%$ & $5 \%$ \\
\hline Mujeres latinas & $6 \%$ & $69 \%$ & $25 \%$ & $6 \%$ \\
\hline Otras & $6 \%$ & $61 \%$ & $31 \%$ & $8 \%$ \\
\hline \multicolumn{5}{|c|}{ Etnia / Edad } \\
\hline Blancos 18-29 años & $12 \%$ & $43 \%$ & $47 \%$ & $10 \%$ \\
\hline Blancos 30-44 años & $16 \%$ & $37 \%$ & $54 \%$ & $19 \%$ \\
\hline Blancos 45-64 años & $30 \%$ & $34 \%$ & $62 \%$ & $4 \%$ \\
\hline Blancos 65 años o más & $13 \%$ & $39 \%$ & $58 \%$ & $17 \%$ \\
\hline Afroamericanos $18-29$ años & $3 \%$ & $85 \%$ & $9 \%$ & $6 \%$ \\
\hline Afroamericanos 30-44 años & $4 \%$ & $89 \%$ & $7 \%$ & $4 \%$ \\
\hline Afroamericanos 45-64 años & $5 \%$ & $90 \%$ & $9 \%$ & $1 \%$ \\
\hline Afroamericanos 65 años o más & $1 \%$ & $91 \%$ & $9 \%$ & $1 \%$ \\
\hline Latinos $18-29$ años & $3 \%$ & $68 \%$ & $26 \%$ & $6 \%$ \\
\hline Latinos 30-44 años & $4 \%$ & $65 \%$ & $28 \%$ & $7 \%$ \\
\hline Latinos $45-64$ años & $4 \%$ & $64 \%$ & $32 \%$ & $4 \%$ \\
\hline Latinos 65 años o más & $1 \%$ & $73 \%$ & $25 \%$ & $2 \%$ \\
\hline Otros & $6 \%$ & $61 \%$ & $31 \%$ & $8 \%$ \\
\hline \multicolumn{5}{|c|}{ Educación } \\
\hline Secundaria o menos & $18 \%$ & $46 \%$ & $51 \%$ & $3 \%$ \\
\hline Estudios de licenciatura & $32 \%$ & $43 \%$ & $51 \%$ & $6 \%$ \\
\hline Graduado de licenciatura & $32 \%$ & $49 \%$ & $44 \%$ & $7 \%$ \\
\hline Posgrado & $18 \%$ & $58 \%$ & $37 \%$ & $5 \%$ \\
\hline \multicolumn{5}{|c|}{ Licenciatura } \\
\hline Graduado & $50 \%$ & $52 \%$ & $42 \%$ & $6 \%$ \\
\hline No graduado & $50 \%$ & $44 \%$ & $51 \%$ & $5 \%$ \\
\hline \multicolumn{5}{|c|}{ Educación / Etnia } \\
\hline Blancos graduados & $37 \%$ & $45 \%$ & $48 \%$ & $7 \%$ \\
\hline Blancos no graduados & $34 \%$ & $29 \%$ & $66 \%$ & $5 \%$ \\
\hline No blancos graduados & $13 \%$ & $72 \%$ & $22 \%$ & $6 \%$ \\
\hline No blancos no graduados & $16 \%$ & $76 \%$ & $20 \%$ & $4 \%$ \\
\hline \multicolumn{5}{|c|}{ Educación / Etnia / Género } \\
\hline Hombres blancos graduados & $17 \%$ & $39 \%$ & $53 \%$ & $8 \%$ \\
\hline Hombres blancos no graduados & $16 \%$ & $23 \%$ & $71 \%$ & $6 \%$ \\
\hline Mujeres blancas graduadas & $20 \%$ & $51 \%$ & $44 \%$ & $5 \%$ \\
\hline Mujeres blancas no graduadas & $17 \%$ & $34 \%$ & $61 \%$ & $5 \%$ \\
\hline No blancos & $29 \%$ & $74 \%$ & $21 \%$ & $5 \%$ \\
\hline \multicolumn{5}{|c|}{ Ingresos económicos } \\
\hline Menos de $\$ 30$ mil al año & $17 \%$ & $53 \%$ & $40 \%$ & $7 \%$ \\
\hline De $\$ 30$ mil a $\$ 49,999$ al año & $19 \%$ & $52 \%$ & $41 \%$ & $7 \%$ \\
\hline De $\$ 50$ mil a $\$ 99,999$ al año & $30 \%$ & $46 \%$ & $49 \%$ & $5 \%$ \\
\hline De $\$ 100$ mil a $\$ 199,999$ al año & $24 \%$ & $47 \%$ & $48 \%$ & $5 \%$ \\
\hline De $\$ 200$ mil a $\$ 249,999$ al año & $4 \%$ & $49 \%$ & $47 \%$ & $4 \%$ \\
\hline De $\$ 250$ mil en adelante al año & $6 \%$ & $46 \%$ & $46 \%$ & $8 \%$ \\
\hline \multicolumn{5}{|c|}{ Identidad partidista } \\
\hline Demócratas & $36 \%$ & $89 \%$ & $8 \%$ & $3 \%$ \\
\hline Republicanos & $33 \%$ & $8 \%$ & $88 \%$ & $4 \%$ \\
\hline Independientes & $31 \%$ & $42 \%$ & $46 \%$ & $12 \%$ \\
\hline
\end{tabular}

nos
4 años
4 años
años
ños o mas $18-2$
os $30-44$
os $45-6$
65 años
años años
licencia
menos

Facultad de Ciencias Políticas, Sociales y de la Comunicación

Universidad de La Laguna

Avenida César Manrique, s/n; Campus de Guajara

38071 La Laguna, Tenerife (Islas Canarias - España) 


\begin{tabular}{|c|c|c|c|c|}
\hline \multicolumn{5}{|c|}{ Preferencia partidista / Género } \\
\hline Hombres Demócratas & $14 \%$ & $87 \%$ & $9 \%$ & $4 \%$ \\
\hline Mujeres Demócratas & $23 \%$ & $91 \%$ & $7 \%$ & $2 \%$ \\
\hline Hombres Republicanos & $17 \%$ & $7 \%$ & $89 \%$ & $4 \%$ \\
\hline Mujeres Republicanas & $16 \%$ & $9 \%$ & $88 \%$ & $3 \%$ \\
\hline Hombres independientes & $17 \%$ & $38 \%$ & $50 \%$ & $12 \%$ \\
\hline Mujeres independientes & $14 \%$ & $47 \%$ & $42 \%$ & $11 \%$ \\
\hline \multicolumn{5}{|c|}{ Ideología } \\
\hline Liberal & $26 \%$ & $84 \%$ & $10 \%$ & $6 \%$ \\
\hline Moderado & $39 \%$ & $52 \%$ & $40 \%$ & $8 \%$ \\
\hline Conservador & $35 \%$ & $16 \%$ & $81 \%$ & $3 \%$ \\
\hline \multicolumn{5}{|c|}{ Estado civil } \\
\hline Casados & $59 \%$ & $44 \%$ & $52 \%$ & $4 \%$ \\
\hline No casados & $41 \%$ & $55 \%$ & $37 \%$ & $8 \%$ \\
\hline \multicolumn{5}{|c|}{ Estado civil / Género } \\
\hline Hombres casados & $29 \%$ & $38 \%$ & $57 \%$ & $5 \%$ \\
\hline Mujeres casadas & $30 \%$ & $49 \%$ & $47 \%$ & $4 \%$ \\
\hline Hombres no casados & $18 \%$ & $46 \%$ & $44 \%$ & $10 \%$ \\
\hline Mujeres no casadas & $23 \%$ & $63 \%$ & $32 \%$ & $5 \%$ \\
\hline \multicolumn{5}{|c|}{ Religión } \\
\hline Protestante & $27 \%$ & $36 \%$ & $59 \%$ & $5 \%$ \\
\hline Católico & $23 \%$ & $46 \%$ & $50 \%$ & $4 \%$ \\
\hline Mormón & $1 \%$ & $28 \%$ & $56 \%$ & $16 \%$ \\
\hline Otra creencia católica & $24 \%$ & $43 \%$ & $54 \%$ & $3 \%$ \\
\hline Judío & $3 \%$ & $71 \%$ & $23 \%$ & $6 \%$ \\
\hline Musulmán & $1 \%$ & $\mathrm{n} / \mathrm{a}$ & $\mathrm{n} / \mathrm{a}$ & $\mathrm{n} / \mathrm{a}$ \\
\hline Otra religión & $7 \%$ & $58 \%$ & $32 \%$ & $10 \%$ \\
\hline No religión & $15 \%$ & $67 \%$ & $25 \%$ & $8 \%$ \\
\hline \multicolumn{5}{|c|}{ Participación en servicios religiosos } \\
\hline Semanalmente o más & $33 \%$ & $41 \%$ & $55 \%$ & $4 \%$ \\
\hline Más de una vez por mes & $16 \%$ & $47 \%$ & $49 \%$ & $4 \%$ \\
\hline Cada mes & $49 \%$ & $43 \%$ & $53 \%$ & $4 \%$ \\
\hline Seguidamente durante el año & $29 \%$ & $48 \%$ & $46 \%$ & $6 \%$ \\
\hline Pocas veces por año & $51 \%$ & $54 \%$ & $39 \%$ & $7 \%$ \\
\hline Nunca & $22 \%$ & $62 \%$ & $30 \%$ & $8 \%$ \\
\hline \multicolumn{5}{|c|}{ Agrupación sindical } \\
\hline Con afiliación & $18 \%$ & $51 \%$ & $42 \%$ & $7 \%$ \\
\hline Sin afiliación & $82 \%$ & $46 \%$ & $48 \%$ & $6 \%$ \\
\hline \multicolumn{5}{|c|}{ Servicio en el ejército } \\
\hline Veterano & $13 \%$ & $34 \%$ & $60 \%$ & $6 \%$ \\
\hline No veterano & $87 \%$ & $50 \%$ & $44 \%$ & $6 \%$ \\
\hline \multicolumn{5}{|c|}{ Nacimiento en el país } \\
\hline Nacido en el país & $91 \%$ & $45 \%$ & $49 \%$ & $6 \%$ \\
\hline Inmigrante & $9 \%$ & $64 \%$ & $31 \%$ & $5 \%$ \\
\hline \multicolumn{5}{|c|}{ Votante por primera vez } \\
\hline Por primera vez & $10 \%$ & $57 \%$ & $38 \%$ & $5 \%$ \\
\hline Segunda ocasión o más & $90 \%$ & $47 \%$ & $47 \%$ & $6 \%$ \\
\hline
\end{tabular}

Facultad de Ciencias Políticas, Sociales y de la Comunicación Universidad de La Laguna 


\begin{tabular}{|c|c|c|c|c|}
\hline \multicolumn{5}{|c|}{ Tiempo de decisión de voto } \\
\hline Últimos días & $8 \%$ & $43 \%$ & $43 \%$ & $14 \%$ \\
\hline Última semana & $5 \%$ & $41 \%$ & $49 \%$ & $10 \%$ \\
\hline En octubre & $12 \%$ & $37 \%$ & $51 \%$ & $12 \%$ \\
\hline En septiembre & $12 \%$ & $46 \%$ & $48 \%$ & $6 \%$ \\
\hline Antes de septiembre & $60 \%$ & $52 \%$ & $45 \%$ & $3 \%$ \\
\hline \multicolumn{5}{|c|}{ Opinión sobre los candidatos presidenciales } \\
\hline Opinión favorable & $41 \%$ & $53 \%$ & $41 \%$ & $6 \%$ \\
\hline Opinión reservada & $32 \%$ & $49 \%$ & $48 \%$ & $3 \%$ \\
\hline Desagrado hacia el oponente & $25 \%$ & $39 \%$ & $50 \%$ & $11 \%$ \\
\hline \multicolumn{5}{|c|}{ Tema más importante para el país } \\
\hline Política exterior & $13 \%$ & $60 \%$ & $33 \%$ & $7 \%$ \\
\hline Inmigración & $13 \%$ & $33 \%$ & $64 \%$ & $3 \%$ \\
\hline Economía & $52 \%$ & $52 \%$ & $41 \%$ & $7 \%$ \\
\hline Terrorismo & $18 \%$ & $40 \%$ & $57 \%$ & $3 \%$ \\
\hline \multicolumn{5}{|c|}{ Candidato que ofrece más alternativas } \\
\hline Puede ayudarme & $15 \%$ & $57 \%$ & $34 \%$ & $9 \%$ \\
\hline Puede generar cambios & $39 \%$ & $14 \%$ & $82 \%$ & $4 \%$ \\
\hline Experiencia & $22 \%$ & $90 \%$ & $7 \%$ & $3 \%$ \\
\hline Justicia & $20 \%$ & $65 \%$ & $25 \%$ & $10 \%$ \\
\hline \multicolumn{5}{|c|}{ Trabajo de los inmigrantes indocumentados } \\
\hline Ofrece status legal & $70 \%$ & $61 \%$ & $33 \%$ & $6 \%$ \\
\hline Ofrece deportación & $25 \%$ & $14 \%$ & $83 \%$ & $3 \%$ \\
\hline \multicolumn{5}{|c|}{ Muro en la frontera sur } \\
\hline A favor & $41 \%$ & $10 \%$ & $85 \%$ & $5 \%$ \\
\hline En contra & $54 \%$ & $76 \%$ & $16 \%$ & $8 \%$ \\
\hline \multicolumn{5}{|c|}{ Efectos sobre el libre comercio } \\
\hline Creación de trabajos & $39 \%$ & $59 \%$ & $35 \%$ & $6 \%$ \\
\hline Pérdida de trabajos & $42 \%$ & $32 \%$ & $64 \%$ & $4 \%$ \\
\hline Sin efecto & $11 \%$ & $65 \%$ & $30 \%$ & $5 \%$ \\
\hline \multicolumn{5}{|c|}{ Guerra contra el Estado Islámico } \\
\hline Va muy bien & $6 \%$ & $85 \%$ & $11 \%$ & $4 \%$ \\
\hline Va extraordinariamente bien & $35 \%$ & $71 \%$ & $24 \%$ & $5 \%$ \\
\hline Va extraordinariamente mal & $28 \%$ & $37 \%$ & $55 \%$ & $8 \%$ \\
\hline Va muy mal & $24 \%$ & $12 \%$ & $83 \%$ & $5 \%$ \\
\hline \multicolumn{5}{|c|}{ Suprema Corte como garante del voto } \\
\hline Factor muy importante & $21 \%$ & $41 \%$ & $56 \%$ & $3 \%$ \\
\hline Factor importante & $48 \%$ & $49 \%$ & $46 \%$ & $5 \%$ \\
\hline Factor de menor importancia & $14 \%$ & $49 \%$ & $40 \%$ & $11 \%$ \\
\hline Factor nulo & $14 \%$ & $55 \%$ & $37 \%$ & $8 \%$ \\
\hline \multicolumn{5}{|c|}{ Equidad del sistema de justicia penal } \\
\hline Trato igual a todos & $43 \%$ & $23 \%$ & $73 \%$ & $4 \%$ \\
\hline Trato inequitativo a afroamericanos & $48 \%$ & $72 \%$ & $22 \%$ & $6 \%$ \\
\hline \multicolumn{5}{|c|}{ Sistema de Seguridad Social Presidente Obama } \\
\hline No fue suficientemente lejos & $30 \%$ & $78 \%$ & $18 \%$ & $4 \%$ \\
\hline Fue correcto & $18 \%$ & $83 \%$ & $10 \%$ & $7 \%$ \\
\hline Fue demasiado lejos & $47 \%$ & $13 \%$ & $82 \%$ & $5 \%$ \\
\hline
\end{tabular}

Facultad de Ciencias Políticas, Sociales y de la Comunicación Universidad de La Laguna 


\begin{tabular}{|c|c|c|c|c|}
\hline \multicolumn{5}{|c|}{ Opinión sobre el Gobierno Federal } \\
\hline Entusiasta & $6 \%$ & $78 \%$ & $19 \%$ & $3 \%$ \\
\hline Satisfecho & $24 \%$ & $76 \%$ & $19 \%$ & $5 \%$ \\
\hline Insatisfecho & $46 \%$ & $46 \%$ & $48 \%$ & $6 \%$ \\
\hline Molesto & $23 \%$ & $18 \%$ & $75 \%$ & $7 \%$ \\
\hline \multicolumn{5}{|c|}{ Opinión sobre la acción del Gobierno } \\
\hline Pudo haber hecho más & $45 \%$ & $74 \%$ & $22 \%$ & $4 \%$ \\
\hline Hizo bastante & $50 \%$ & $22 \%$ & $72 \%$ & $4 \%$ \\
\hline \multicolumn{5}{|c|}{ Opinión sobre Barack Obama como presidente } \\
\hline Alto nivel de aprobación & $33 \%$ & $93 \%$ & $4 \%$ & $3 \%$ \\
\hline Buen nivel de aprobación & $20 \%$ & $69 \%$ & $20 \%$ & $11 \%$ \\
\hline Buen nivel de desaprobación & $12 \%$ & $14 \%$ & $77 \%$ & $9 \%$ \\
\hline Alto nivel de desaprobación & $33 \%$ & $3 \%$ & $93 \%$ & $4 \%$ \\
\hline \multicolumn{5}{|c|}{ Condiciones del nuevo presidente } \\
\hline Continuar políticas de Obama & $28 \%$ & $91 \%$ & $5 \%$ & $4 \%$ \\
\hline Más conservador que Obama & $47 \%$ & $13 \%$ & $82 \%$ & $5 \%$ \\
\hline Más liberal que Obama & $17 \%$ & $69 \%$ & $23 \%$ & $8 \%$ \\
\hline \multicolumn{5}{|c|}{ Opinión sobre Hillary Clinton } \\
\hline Favorable & $43 \%$ & $96 \%$ & $3 \%$ & $1 \%$ \\
\hline Desfavorable & $55 \%$ & $11 \%$ & $81 \%$ & $8 \%$ \\
\hline \multicolumn{5}{|c|}{ Opinión sobre Donald Trump } \\
\hline Favorable & $38 \%$ & $4 \%$ & $95 \%$ & $1 \%$ \\
\hline Desfavorable & $60 \%$ & $77 \%$ & $15 \%$ & $8 \%$ \\
\hline \multicolumn{5}{|c|}{ Opinión sobre Hillary Clinton y Donald Trump } \\
\hline Favorable para ambos & $2 \%$ & $\mathrm{~N} / \mathrm{A}$ & $\mathrm{N} / \mathrm{A}$ & - \\
\hline Favorable a Clinton & $41 \%$ & $98 \%$ & $1 \%$ & $1 \%$ \\
\hline Favorable a Trump & $36 \%$ & $1 \%$ & $98 \%$ & $1 \%$ \\
\hline Desfavorable para ambos & $18 \%$ & $30 \%$ & $47 \%$ & $23 \%$ \\
\hline
\end{tabular}

\section{Valor simbólico de los candidatos}

El empresario Donald Trump de 68 años ${ }^{15}$ alcanzó la nominación Republicana sin contar con antecedentes partidarios, se adentró en la esfera política mediante una campaña independiente contra el presidente Obama en 2012 al que acusó de no presentar su verdadero certificado de nacimiento para poder acceder a la Presidencia. Posteriormente, en un anuncio pagado cuestionó a la élite política estadounidense y señaló que, basándose en

\footnotetext{
${ }^{15}$ Con su triunfo se convirtió en el presidente con mayor edad en la historia de Estados Unidos.
} 
su experiencia de empresario exitoso y desligado de la política, podría reorientar el rumbo del país. Durante la precampaña Republicana de 2016 sus críticas constantes al sistema de inmigración y los grupos indocumentados procedentes de México y América Latina desataron una oleada de críticas en muchos sectores, pero también un amplio apoyo por parte de grupos blancos, conservadores y marcadamente religiosos. En esa dimensión, en la elección presidencial no le afectó perder 2.5 millones de sufragios en los estados mayoritariamente Demócratas porque su fuerza de marca se centró más en los territorios indecisos y de voto variable, como Pensilvania, Michigan y Wisconsin. Ello fue posible por su constante manifestación de dudas sobre la capacidad ejecutiva de Hillary Clinton a quien por distintas razones acusó por un “desempeño cuestionable” y amenazó con encarcelarla. Con base en su fuerza discursiva y actitud desenfadada Trump posicionó mejor su imagen de hombre firme y decidido, mientras que Clinton demostró vulnerabilidad y marcadas formas de debilidad, así como la falta de una propuesta capaz de resistir las dudas en los ciudadanos en momentos previos a la elección.

Pese a que su propuesta política pudo haber sido más sólida y extensa, Clinton posicionó lemas que no fueron convergentes entre sí. La frase "I'm with Her" (Estoy con ella) se centró en su imagen personal y, por tanto, resultó débil para identificarse con grupos sociales, culturales y económicos diversos. Ello fue identificado por las encuestas, las que la reportaron como una de las candidatas menos populares de todos los tiempos. Adicionalmente, la frase busco dar una nueva naturaleza histórica a la candidata Clinton como primera dama, política destacada y única mujer nominada por un partido importante a la presidencia del país. Aunque para algunos sectores eso le otorgó una muy buena reputación y alto grado de confianza, para otros -misóginos o antifeministas- su trayectoria llena de claro obscuros y una campaña poco propositiva nunca ofreció elementos de originalidad. Para las condiciones de competencia, su propuesta no resultó atractiva para votantes con un nuevo nivel de expectativa y deseosos de una mayor intervención estatal en 
la economía. En tal forma, su compromiso débil con causas sociales importantes, como la inmigración, la igualdad de géneros o la recuperación del empleo, propició que su imagen como candidata fuera susceptible a casi cualquier ataque y vulnerable a muchos ataques sobre su ética personal y familiar.

La frase "Stronger together" si bien animó a los partidarios de Clinton a identificarse con su nombre, fue en sentido estricto una reiteración de apoyo al grupo en el poder. Sin embargo, ellas fueron duramente criticadas por Trump a quienes calificó de "élites costeras". Para algunos votantes Demócratas esas críticas pudieron haber influido en su percepción y decidido votar por el candidato opositor al considerarlo más sensible de sus necesidades. Clinton ya tenía un problema anterior de imagen, al ser percibida como una política fría y dura para conectar con prioridades políticas dispares. Tal fue el caso de frases como "Queremos preservar Obamacare porque amamos a nuestras familias y vecinos y queremos que sean saludables". "Queremos una política más inteligente del crimen porque amamos a la gente de todas las razas". "Queremos una política de defensa fuerte y cautelosa porque amamos a nuestro país". Ellas fueron constantes en sus discursos, pero carecieron de una significación que conectara sus propuestas políticas con los diferentes segmentos de votantes Demócratas y les otorgara un componente de identificación. En contraste, "Make America great again" del candidato Trump no puso un énfasis en la persona, pero si construyó, como lo hizo Obama en 2008 y 2012, una aspiración mayormente común a todos los ciudadanos con posibilidades de voto. Las palabras más usadas por Trump en sus tuits de promoción fueron great (grande), winner (ganador) o winners (ganadores). En los de ataque, contraste o descalificación, loser (perdedor) o losers (perdedores).

\section{Conclusiones y discusión}

En cualquier otra democracia del mundo Hillary Clinton habría sido designada en 2016 como Presidenta de Estados Unidos. Sin embargo, en un sistema de elección descentralizado e 
indirecto como el vigente la mayor cantidad de votos populares no garantiza necesariamente el triunfo final. Desde la fundación del país por definición constitucional mucho se ha argumentado si es el modelo electoral más pertinente para preservar el federalismo y no permitir que los estados con mayor población impongan su mayoría. Sin embargo, ello no ha resultado así porque en la práctica los amplios márgenes de votación que obtiene el Partido Demócrata en California y el noreste (Nueva York, Connecticut y Massachusetts) no han sido suficientes para obtener ventaja en el voto popular. Tampoco es cierto que el sistema del colegio electoral preserva el federalismo, pues la institución prevista para ello es el Senado. De hecho, el sistema del colegio electoral buscaba garantizar que la Presidencia de los primeros años postrevolucionarios no saliera de las élites de Virginia y Massachusetts. Sin embargo, de los primeros seis presidentes cuatro eran originarios de Virginia (Washington, Jefferson, Madison y Monroe) y dos de Massachusetts (John Adams y su hijo John Quincy).

Incluso varias veces en la historia el voto mayoritario no ha servido para elegir al presidente del país en el sistema del colegio electoral. En la era moderna esto ha ocurrido dos veces, una para imponer sobre el voto popular a George W Bush en 2000 (tras una impugnación de los resultados electorales en La Florida que lo favorecieron) y en 2016 a Donald Trump. La experiencia de las presidencias sin el favor del voto popular nunca ha sido buena, o al menos han resultado controversiales y de un solo periodo. Es lo que ocurrió en el pasado con el propio George Bush, quien pudo reelegirse para la Presidencia, pero fue quizás una de las administraciones más opacas de la era moderna con un saldo infructuoso por el gran error de la Guerra en Irak y la recesión económica más grave experimentada por el país desde la Gran Depresión. En ese sentido, si bien las condiciones de la elección 2016 le fueron favorables la experiencia histórica no generaría buenos augurios para la continuidad del gobierno de Trump por dos periodos. 
- Primero, porque si bien nadie cuestiona la constitucionalidad de la elección, se mantiene la discusión sobre la viabilidad y pertinencia del sistema de elección presidencial por voto indirecto

- Segundo, porque Trump asumió una presidencia sin mandato programático claro. En tal forma, debe señalarse que la mayoría que apoya sus ideas violentas altera el sentido mismo de los valores democráticos y la soberanía popular. Ello hace prever tensiones sociales y una presidencia de solo cuatro años.

- Tercero, porque Trump ganó como expresión del elector blanco, con menor nivel de educación y más conservatismo religioso. Perdió, sin embargo, el voto de las mujeres, los latinos, los afroamericanos y los jóvenes, quienes claman hoy por una visión más incluyente que la que existe en los sectores rurales.

Además de las tensiones raciales y de orden social que planteó la agenda de Trump, su denuncia abierta a los tratados de libre comercio fue fundamental para la victoria. Ese es uno de los asuntos que más lo distancia de la plataforma Republicana en ambas cámaras del Congreso y es mayoritariamente proclive a ellos. Por su parte, el Partido Demócrata tiene una fisura grande que deberá resolver en el futuro a partir de esos resultados. ¿La visión de Obama o la de Sanders? Asimismo, los miedos y las tensiones desatadas por la retórica de la polarización enfrentan ya en los lugares público a la población anglosajona con los latinos, afroamericanos, musulmanes americanos y las parejas o matrimonios gay.

\section{Referencias}

Aglesta, Jennifer. Encuesta de encuestas de CNN: Hillary Clinton aventaja a Donald Trump en 10 puntos, en http://cnnespanol.cnn.com/2016/08/08/encuesta-de-encuestas-decnn-hillary-clinton-aventaja-a-donald-trump-en-10-puntos/.

American Association for Public Opinion Researchers. Statement on Trump/Pence Campaign Web Survey, en http://www.aapor.org/Publications-Media/Public-Statements/AAPORStatement-on-Trump-Pence-Campaign-Web-Survey.aspx 
Bárbaro, Miguel. Conciso, mezquino y poderoso: cómo Donald Trump dominó Twitter en 2016, en The New York Times, 5 de octubre de 2017. https://www.nytimes.com/2015/10/06/us/politics/donald-trump-twitter-use-campaign2016.html

BBC Mundo. ¿Por qué Hillary Clinton perdió las elecciones en EE.UU. pese a conseguir 2,8 millones de votos más que Donald Trump?. Washington, 26 de enero de 2017, http://www.bbc.com/mundo/noticias-internacional-37933771

Beltrán, Daniel. Redes sociales virtuales como dispositivos mediáticos contemporáneos, en Cuadernos de Lingüística Hispánica No 30. Julio - diciembre de 2017, pp. 105-123. http://www.scielo.org.co/pdf/clin/n30/0121-053X-clin-30-00105.pdf

Cellán, Rory. Elecciones en Estados Unidos: ¿fue Facebook la clave para el triunfo de Donald Trump?, en BBC News, 11 noviembre 2016. https://www.bbc.com/mundo/noticias-internacional-37946548

Chadwick, Andrew (2013). The Hybrid Media System: Politics and Power. Oxford: Oxford University Press.

Clementson, David, Pascual-Ferrá, Paola \& Beatty, Michael. ¿Cuándo un candidato presidencial parece presidencial y digno de confianza? Mensajes de campaña a través del lente de la teoría de la expectativa del lenguaje, in Presidential Studies Quarterly Num. 3. Volume LCVI. Center for the Study of the Presidency and Congress. September, 2016. pp. 592-617.

Del Tronco, José, Flores Georgina y Madrigal, Abby. La utilidad de las encuestas en la predicción del voto. La segunda vuelta de Argentina 2015, en Revista Mexicana de Opinión Pública, julio - diciembre de 2016. pp. 73 - 92. http://www.scielo.org.mx/pdf/rmop/n21/2448-4911-rmop-21-00073.pdf

Díez, Beatriz. Elecciones en Estados Unidos: 5 razones que explican la (quizá no tan) sorprendente victoria de Donald Trump sobre Hillary Clinton, en BBC News. Los Ángeles, 9 de noviembre de 2016. https://www.bbc.com/mundo/noticias-internacional$\underline{37917754}$

Drobnic, Angie. Mentira del año 2016: noticias falsas, en POLITIFACT, 13 de diciembre de 2016. Instituto Pointer. https://www.politifact.com/article/2016/dec/13/2016-lie-yearfake-news/

Diario.es. El rastreador. Las frases más salvajes del presidente Donald Trump. http://www.eldiario.es/rastreador/frases-estupidas-Donald-Trump 6 478112190.html

Fajardo, Luis. La elección de Hillary Clinton significaría el fin de la libertad de los estadounidenses: Radiografía de los votantes de Donald Trump, en BBC News 28 de octubre de 2016. https://www.bbc.com/mundo/noticias-internacional-37791553 
Faus, Joan. El FBI investiga nuevos correos privados de Hillary Clinton. Los documentos proceden de un caso separado sobre el marido de una asesora de la demócrata, en Periódico El País. Washington, 29 de octubre de 2016. https://elpais.com/internacional/2016/10/28/estados unidos/1477675819923042.html

Federal Election Commision. Federal Elections 2016. Election Results for the U S President, the U S Senate and U S House Representatives. Washington, D. C.

Federal Election Commision. Federal Elections 2012. Election Results for the U S President, the U S Senate and U S House Representatives. Washington, D. C.

Federal Election Commission. Federal Elections 2008. Election Results for the U S President, the U S Senate and U S House Representatives. Washington, D. C.

Fry, R. Millennials and Gen Xers outvoted Boomers and Older Generations in 2016 election. Pew Research Center. Washington, julio 31 de 2017. http://www.pewresearch.org/facttank/2017/07/31/millennials-and-gen-xers-outvoted-boomers-and-older-generations-in2016-election/.

Faus, Joan. El FBI investiga nuevos correos privados de Hillary Clinton. Los documentos proceden de un caso separado sobre el marido de una asesora de la demócrata, en Periódico El País. Washington, 29 de octubre de 2016. https:/elpais.com/internacional/2016/10/28/estados unidos/1477675819 923042.html

Gallup News. Presidential Election 2016: Key Indicators, en http://news.gallup.com/poll/189299/presidential-election-2016-key-indicators.aspx.

Pew Research Center. Cubriendo al presidente Trump en un entorno mediático polarizado. Washington, 2 de octubre de 2017. https://www.journalism.org/2017/10/02/trump-first100-days-methodology/

Pew Research Center. Para la mayoría de los votantes de Trump. Los sentimientos muy cálidos por el perduraron. Un examen del electorado de 2016, basado en votantes validados. Washington, 9 de agosto de 2016. https://www.pewresearch.org/politics/2018/08/09/an-examination-of-the-2016electorate-based-on-validated-voters/

Pew Research Center. La brecha generacional y las elecciones de 2012. Visión general. 3 de agosto de noviembre de 2011. https://www.pewresearch.org/politics/2011/11/03/thegeneration-gap-and-the-2012-election-3/

Rodríguez, Roberto. (2018). Trump 2016: ¿presidente gracias a las redes sociales?, en Revista Palabra Clave No 21. Vol. 3. pp, 831-859. http://www.scielo.org.co/pdf/pacla/v21n3/0122-8285-pacla-21-03-00831.pdf

Social Flow. Trump tuvo tres veces más exposición gratuita en redes sociales que Hillary Clinton lo que lo convirtió rápidamente en la persona de la que más se habló en el

Facultad de Ciencias Políticas, Sociales y de la Comunicación Universidad de La Laguna 
planeta. Elecciones en Ustados Unidos 2016. https://www.facebook.com/SocialFlowMx/

The American Presidency Project. Election Results for the US President, 2016. en http://www.presidency.ucsb.edu/showelection.php?year=2016

Ward, Ch., Welsh, B., Conley, A. \& Smith, P. J. It's About Time: Examining the Effect of Interviewer-Quoted Survey Completion Time Estimates on Survey Efficiency, en Survey Practice No. 2. Volumen No. 10.2017. http://www.surveypractice.org/index.php/SurveyPractice/article/view/374/pdf 91.

The New York Times (2017). Michigan Presidential Race. Results: Donald J. Trump Wins https://www.nytimes.com/elections/results/michigan-president-clinton-trump.

\section{Forma de citar este artículo en bibliografías}

MEYER, J.A. (2017): "Entre la polarización y la catarsis: Nuevas directrices del comportamiento electoral en Estados Unidos", en Revista PANGEA N 8, páginas 20 a 48. Tenerife: Red Académica Iberoamericana de Comunicación. Recuperado el _ de de 2 de: http://www.revistapangea.org 